\section{Legalización de muerte médicamente asistida: discusión sobre el potencial impacto en el desarrollo de cuidados paliativos}

\author{
MARIANA DITTBORN B. ${ }^{1}$, CONSTANZA MICOLICH V. ${ }^{2,3}$
}

\section{Legalization of medically assisted death: its potential impact on the development of palliative care}

In Chile, there are four bills to legalize euthanasia, an act which public surveys report as supported by most of the population. At the legislative, healthcare and social level, there is an active debate about euthanasia, the rights of terminally ill patients and the context of Palliative Care (PC) in the country. Chilean literature on euthanasia focuses mainly on the ethical analysis of the act itself but does not address the moral legitimacy of the legalization of this practice. This distinction is relevant since the probity of a particular action does not determine the moral legitimacy of its implementation at a public policy level. One aspect of this dimension is the potential negative impact of the legalization of physician-assisted death (PAD) on the development of PC services. This issue is particularly relevant in Chile, where PC provision is currently suboptimal and mostly restricted to cancer patients. This paper analyses available evidence on the potential impairment of PC development after PAD legalization. Although the analysis of evidence has some limitations, this concern is not supported by the available evidence. However, any project about PAD legalization must contemplate a factual commitment with the development of minimum PC provision, according to international recommendations.

(Rev Med Chile 2020; 148: 665-673)

Key words: Euthanasia; Palliative Care; Palliative Medicine; Suicide, Assisted.

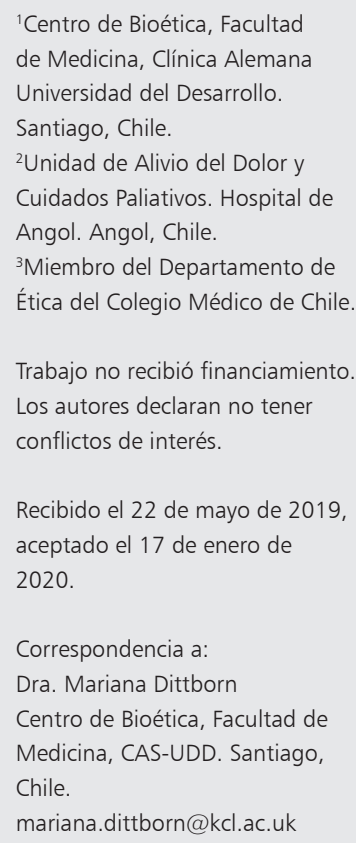

Recibido el 22 de mayo de 2019 , aceptado el 17 de enero de 2020.

Correspondencia a:

Dra. Mariana Dittborn

Centro de Bioética, Facultad de Medicina, CAS-UDD. Santiago,

Chile.

mariana.dittborn@kcl.ac.uk

E n Chile, desde 2011, existen cuatro proyectos de ley relativos a la eutanasia en tramitación en la Cámara de Diputados del Congreso Nacional ${ }^{1}$. Paralelamente, una encuesta reciente de la CADEM $(2018)^{2}$ reportó que $67 \%$ de los participantes estaba de acuerdo con la eutanasia y la encuesta a médicos pertenecientes al Colegio Médico de Chile (2019) arrojó que 77\% estaría de acuerdo con la misma ${ }^{3}$. Estos hechos han generado un debate a nivel legislativo, médico y ciudadano ${ }^{4}$, no solo en torno a la propia eutanasia, sino sobre todo acerca de los derechos de las personas con enfermedades terminales y la situación de los cuidados paliativos $(\mathrm{CP})$ en Chile.

La literatura internacional sobre los argumentos éticos a favor o en contra de la muerte médicamente asistida (MMA) es amplia, sin existir consenso. En nuestro medio, la discusión se ha basado principalmente en el análisis ético-moral de MMA, presentándose argumentos a favor ${ }^{5-7} \mathrm{O}$ en contra de la misma ${ }^{8-12}$. Sin embargo, la discusión sobre la legitimidad moral de la legalización 
de MMA ha sido abordada parcialmente $e^{6,13}$. Esta distinción es relevante, ya que la probidad de una acción particular no necesariamente determina la corrección moral de su implementación a nivel de política pública ${ }^{13-16}$. En general, el debate legal de MMA se ha caracterizado como un conflicto entre el principio de autonomía versus el deber del Estado de proteger el derecho a la vida de sus ciudadanos ${ }^{4,17}$. Una deliberación comprehensiva debe incluir, además, las posibles consecuencias de la legalización para la sociedad en su conjunto, ponderando la autonomía individual frente a otros intereses sociales relevantes ${ }^{6,18-20}$.

Si bien el análisis ético sobre MMA es esencial en el debate, lo que motiva este trabajo es el abordaje de la legitimidad moral de la legalización de MMA, considerando particularmente el potencial impacto de esta en el desarrollo de CP.

\section{Definiciones}

Eutanasia, del griego eu-thanasía, significa literalmente buena muerte. Sin embargo, no hay consenso sobre su definición, planteándose variados términos y neologismos desde diferentes perspectivas $^{21-23}$. Esta ambigüedad en los términos ha llevado muchas veces a supuestos desacuerdos morales cuando no necesariamente los hay, siendo esencial clarificar los conceptos ${ }^{24,25}$. En este trabajo se tomarán como referencia las definiciones consensuadas por la Asociación Europea de Cuidados Paliativos (EAPC, por sus siglas en inglés "European Association for Palliative Care") ${ }^{26}$, que se describen en la Tabla 1. Para efectos de este trabajo, el concepto de MMA incluye ambas prácticas, eutanasia y suicidio médicamente asistido (SMA), acorde a la terminología ampliamente utilizada en investigación relacionada ${ }^{14,27-30}$. Si bien existen considerables diferencias conceptuales y éticas entre estas prácticas ${ }^{31}$, estas no son relevantes para el argumento central a discutir en este trabajo.

\section{Contexto global}

A nivel internacional, existen 5 países con legalización de MMA, mientras que Suiza y 5 estados de Estados Unidos de Norteamérica (EE. UU.) permiten únicamente el SMA.

En la Tabla 2 se enumeran aquellos países con legalización de MMA y el nivel de desarrollo de CP según el atlas de la EAPC de 2014²,35.

\section{Contexto chileno}

En 2012 entró en acción la Ley 20.584 que regula los derechos y deberes que tienen las personas en relación con acciones vinculadas a su atención en salud ${ }^{36}$. Dos artículos de esta ley son relevantes para la discusión sobre MMA, detallados en la Tabla 3.

Estos artículos han sido ampliamente discutidos por académicos debido a su ambigüedad e inutilidad como marco para las decisiones médicas de final de vida $a^{5,7,17,37-39}$. La ley no aporta una definición precisa de lo que incluyen las "prácticas eutanásicas" ni "medidas de soporte ordinario". Además, parece inconducente que un rechazo a tratamiento implique una "aceleración artificial de la muerte". Por otra parte, se explicita el derecho a CP a las personas con enfermedades terminales, mas solo existen garantías de aquello para los pacientes con enfermedades oncológicas.

En 1995 se creó el Programa Nacional para el Alivio del Dolor y Cuidados Paliativos y en 2003 se instauró como garantía explícita de salud (GES) el acceso a alivio del dolor y CP para pacientes con cáncer avanzado ${ }^{40}$. Luego, en 2015, se extendió el tratamiento integral por alivio del dolor a pacientes con diagnóstico oncológico, independiente de la etapa en que se encuentren ${ }^{41}$. Actualmente, se encuentra en trámite constitucional en la Cámara de Diputados el proyecto de ley que regula el "Reconocimiento y protección de los derechos de las personas con enfermedades terminales, y el buen morir", el cual extendería la cobertura a pacientes con enfermedades terminales no oncológicas, pero no considera, explícitamente, financiamiento para la implementación de los derechos mencionados ${ }^{42}$.

Se agrega a esta situación la existencia de un sistema de salud segmentado público/privado, cuyo modelo genera gran inequidad, manteniendo un sistema público carente de recursos humanos y estructurales necesarios para satisfacer las necesidades de la población, afectando principalmente a la población más pobre ${ }^{43}$. Paradójicamente, Chile está clasificado por el Atlas Global de Cuidados Paliativos de la Organización Mundial de la Salud (OMS) (2014) ${ }^{35}$ dentro de los países que presentan servicios de CP con integración avanzada con otros servicios del sistema nacional de salud. 
Tabla 1. Glosario de términos según definiciones de EAPC

\begin{tabular}{|c|c|c|}
\hline Concepto & Definición & Comentarios \\
\hline Eutanasia & $\begin{array}{l}\text { La muerte producida intencionalmente por un/a } \\
\text { médico/a mediante la administración de drogas, } \\
\text { a una persona que lo ha solicitado de manera } \\
\text { competente y voluntaria }\end{array}$ & $\begin{array}{l}\text { La eutanasia es, por definición, siempre activa } \\
\text { y bajo la solicitud voluntaria de la persona a la } \\
\text { que se le aplicará }\end{array}$ \\
\hline $\begin{array}{l}\text { Eutanasia no } \\
\text { voluntaria }\end{array}$ & $\begin{array}{l}\text { Terminar con la vida de un paciente sin su solici- } \\
\text { tud expresa cuando este no tiene capacidad para } \\
\text { consentir (paciente no competente) }\end{array}$ & \\
\hline $\begin{array}{l}\text { Eutanasia } \\
\text { involuntaria }\end{array}$ & $\begin{array}{l}\text { Terminar con la vida de un paciente competente } \\
\text { sin su solicitud expresa, en contra de su voluntad } \\
\text { o sin su consentimiento. }\end{array}$ & $\begin{array}{l}\text { como "Término de la vida sin la solicitud } \\
\text { explicita del paciente (TVSSE) }{ }^{22}\end{array}$ \\
\hline Suicidio asistido & $\begin{array}{l}\text { Cuando una persona asiste intencionalmente a } \\
\text { otra persona a terminar con su vida, previo a una } \\
\text { solicitud voluntaria y competente }\end{array}$ & \\
\hline $\begin{array}{l}\text { Suicidio } \\
\text { médicamente } \\
\text { asistido }\end{array}$ & $\begin{array}{l}\text { Cuando un/a médico/a asiste intencionalmente } \\
\text { a una persona a terminar con su vida proveyén- } \\
\text { dole de las drogas necesarias para su autoad- } \\
\text { ministración, previo a una solicitud voluntaria y } \\
\text { competente }\end{array}$ & $\begin{array}{l}\text { Eventualmente sugeriría precisar si se podría } \\
\text { aplicar a otro profesional sanitario no médico el } \\
\text { término "médicamente asistido" }\end{array}$ \\
\hline $\begin{array}{l}\text { Decisiones de no } \\
\text { tratar o limitación } \\
\text { de esfuerzo } \\
\text { terapéutico (LET) }\end{array}$ & $\begin{array}{l}\text { No iniciar o retirar medidas que son consideradas } \\
\text { desproporcionadas o ante el rechazo de trata- } \\
\text { miento del paciente }\end{array}$ & $\begin{array}{l}\text { Considerado legal y ético cuando se cumplen } \\
\text { las condiciones y debe ser siempre discutido con } \\
\text { el paciente o en su defecto con sus cuidadores } \\
\text { Por medidas desproporcionadas se entienden } \\
\text { aquellas en que los beneficios anticipados son } \\
\text { superados por las cargas esperadas }{ }^{32}\end{array}$ \\
\hline $\begin{array}{l}\text { Sedación paliativa } \\
\text { (SP) }\end{array}$ & $\begin{array}{l}\text { El uso monitoreado de medicamentos para inducir } \\
\text { un estado de conciencia disminuido o abolido } \\
\text { (estado de inconciencia) para aliviar la carga de un } \\
\text { sufrimiento que no ha sido posible mediante otras } \\
\text { medidas éticamente aceptables para el paciente, } \\
\text { su familia y el personal de salud }\end{array}$ & $\begin{array}{l}\text { Esto debe ser acordado con el paciente, en su } \\
\text { defecto con sus cuidadores } \\
\text { Esta es una práctica aceptada cuando se uti- } \\
\text { liza en situaciones apropiadas para pacientes } \\
\text { paliativos seleccionados. La intención es lograr } \\
\text { el control de los síntomas y hay evidencia que } \\
\text { demuestra que la SP no acorta la sobrevida en } \\
\text { los pacientes con cáncer avanzado }\end{array}$ \\
\hline $\begin{array}{l}\text { Cuidados } \\
\text { paliativos (CP) }\end{array}$ & $\begin{array}{l}\text { Los cuidados paliativos constituyen un plan- } \\
\text { teamiento que mejora la calidad de vida de los } \\
\text { pacientes (adultos y niños) y sus familiares cuando } \\
\text { afrontan problemas inherentes a una enfermedad } \\
\text { crónica avanzada. Previenen y alivian el sufrimien- } \\
\text { to a través de la identificación temprana, la eva- } \\
\text { luación y el tratamiento correctos del dolor y otros } \\
\text { problemas, sean estos de orden físico, psicosocial } \\
\text { o espiritual }{ }^{34}\end{array}$ & \\
\hline
\end{tabular}


Tabla 2. Contexto global sobre MMA y nivel de desarrollo de CP

\begin{tabular}{|c|c|c|c|}
\hline País o Región & Año de legalización & Figuras legalizadas & $\begin{array}{l}\text { Desarrollo de CP según } \\
\text { Atlas EAPC (2014) }\end{array}$ \\
\hline Países Bajos & 1994 & Eutanasia y SMA & $4 a$ \\
\hline Bélgica & 2002 & Eutanasia y SMA & $4 b$ \\
\hline Luxemburgo & 2009 & Eutanasia y SMA & $4 b$ \\
\hline Colombia & 1997 & Eutanasia y SMA & $3 a$ \\
\hline Canadá & 2016 & Eutanasia y SMA & $4 b$ \\
\hline Oregon, Estados Unidos & 1997 & SMA & $4 b$ \\
\hline Washington, Estados Unidos & 2009 & SMA & \\
\hline Montana, Estados Unidos & 2009 & SMA & \\
\hline Vermont, Estados Unidos & 2013 & SMA & \\
\hline California, Estados Unidos & 2015 & SMA & \\
\hline Suiza & 1942 & $* *$ & $4 b$ \\
\hline
\end{tabular}

aDefiniciones del nivel de desarrollo de CP según el Atlas Global de Cuidados Paliativos. Grupo 1: Sin actividad de cuidados paliativos conocida. Grupo 2: Desarrollo incipiente. Grupo 3a: Provisión aislada/Grupo 3b: Provisión generalizada. Grupo 4a: Integración preliminar con otros servicios del sistema nacional de salud. Grupo 4b: Integración avanzada con otros servicios del sistema nacional de salud. **El código penal suizo solo considera la asistencia al suicidio un crimen si el motivo es descrito como egoísta. No requiere asistencia de un médico. MMA: Muerte médicamente asistida. CP: Cuidados paliativos. SMA: Suicidio médicamente asistido. EAPC: Asociación europea de cuidados paliativos.

\section{Tabla 3. Artículos Ley $\mathbf{2 0 . 5 8 4}$ relevantes en cuidados de final de vida}

Art. 14 "En ningún caso el rechazo a tratamientos podrá tener como objetivo la aceleración artificial de la muerte, la
realización de prácticas eutanásicas o el auxilio al suicidio"
Art. 16 "La persona que fuere informada de que su estado de salud es terminal, tiene derecho a otorgar o denegar
su voluntad para someterse a cualquier tratamiento que tenga como efecto prolongar artificialmente su vida,
sin perjuicio de mantener las medidas de soporte ordinario. En ningún caso, el rechazo de tratamiento podrá
implicar como objetivo la aceleración artificial del proceso de muerte"
"Las personas que se encuentren en este estado tendrán derecho a vivir con dignidad hasta el momento de la
muerte. En consecuencia, tienen derecho a los cuidados paliativos que les permitan hacer más soportables los
efectos de la enfermedad, a la compañía de sus familiares y personas a cuyo cuidado estén y a recibir, cuando
lo requieran, asistencia espiritual"

Chile está experimentando una transición epidemiológica, con un aumento en la prevalencia y tasas de mortalidad asociadas a enfermedades crónicas no transmisibles, incluido el cáncer ${ }^{44}$, una mayor esperanza de vida y una creciente proporción de personas mayores ${ }^{45}$. Se estima que las necesidades de CP corresponden a 49.622 pacientes/año (sin considerar cuidadores), con al menos 50\% de los pacientes que requieren $\mathrm{CP}$ presentando enfermedades no oncológicas, actualmente no garantizados en el sistema de salud (De Allende-Salazar MI, Valdivia G, y Pizarro A.
Ampliando el marco de necesidades de cuidados paliativos en Chile: Una aproximación metodológica. Resumen presentado en el IX Congreso Latinoamericano de Cuidados Paliativos, 2018).

Por último, el deber de promover el desarrollo de CP universales y de excelencia es incuestionable. Considerando, por ejemplo, que la MMA representa $2,9 \%$ y $4,6 \%$ de todas las muertes anuales en Holanda y Bélgica, respectivamente ${ }^{22}$, esta no parece ser una alternativa universal para todos los pacientes en final de vida. Además, los reportes internacionales indican que $70 \%$ de 
las muertes por MMA corresponde a pacientes con diagnóstico de cáncer ${ }^{22}$, lo que implica que un porcentaje importante de pacientes con diagnósticos no oncológicos acceden a MMA, siendo quienes precisamente no cuentan con la garantía de cuidados paliativos en Chile. Por otra parte, la idea de que la provisión de CP elimina por completo las solicitudes de eutanasia ha sido cuestionada, dada la persistencia de la solicitud de MMA en pacientes que reciben $\mathrm{CP}$ de calidad ${ }^{22,46,47}$.

Estos elementos justifican la necesidad de considerar la evidencia disponible sobre los efectos de la legalización de MMA en el desarrollo de CP.

\section{Impacto de la legislación de MMA sobre el desarrollo de CP}

Se han descrito potenciales consecuencias positivas y negativas asociadas a la legalización de MMA. Entre las positivas se incluyen: que quienes desean asistencia en morir podrán obtenerla; la disminución de sufrimiento en la fase de fin de vida; la tranquilidad y seguridad que obtendrán aquellos que sufren y podrían sentirse aliviados al saber que cuentan con la alternativa de una $\mathrm{MMA}^{14}$, el potencial impacto positivo en la comunicación médico-paciente al permitir una discusión abierta sobre el tema ${ }^{48}$ y que la regulación y estandarización de esta práctica disminuiría los riesgos y potenciales abusos sobre los que sufren condiciones terminales que impone una práctica clandestina de $\mathrm{MMA}^{49}$.

Por su parte, las potenciales consecuencias negativas descritas incluyen: el deterioro de la confianza en la relación médico-paciente; la corrosión moral de la sociedad; el riesgo de abuso o presión indebida sobre poblaciones vulnerables; el deslizamiento por la pendiente resbaladiza (argumento que sostiene que una vez aceptada una posición en particular, será extremadamente difícil, o incluso imposible, no aceptar posiciones más extremas ${ }^{50}$, en este caso, el riesgo de que habiendo iniciado esta práctica con eutanasia voluntaria, se derive luego en prácticas TVSSE) y el debilitamiento del compromiso social por desarrollar cuidados de final de vida apropiados ${ }^{13,14,19,51}$. En la discusión chilena, las posiciones políticas se han dividido entre aquellas que consideran que es esencial avanzar en el desarrollo de CP antes de legislar sobre la MMA y quienes están a favor de la legislación de la MMA considerando que con ello se promovería el desarrollo de los CP en el país. Cabe destacar que existe en la literatura un amplio acuerdo de que la falta de CP no debe ser la razón para solicitar MMA $^{52,53}$. Se ha planteado, entonces, que cualquier política relacionada con esto debe incluir el compromiso de garantizar un mínimo esencial de atención, incluidos disponibilidad de CP universales ${ }^{14,54}$. Con respecto a este mínimo esencial, podría considerarse como referencia lo estipulado por la Comisión Lancet en Cuidados Paliativos de 2017, donde se acordó un paquete mínimo esencial que debería ser accesible universalmente para aquellos con graves problemas de salud asociados a enfermedades crónicas avanzadas, oncológicas y no oncológicas. Este incluiría un listado de medicamentos, equipos médicos y recursos humanos para una provisión de CP segura y efectiva en un entorno de atención primaria de salud ${ }^{14}$.

En los Países Bajos, el desarrollo de CP se produjo en un contexto en el que la MMA estaba ya aceptada (legalizado en 1997), sin acuerdo sobre si la falta de CP explica la legalización de la MMA o viceversa ${ }^{55}$. En Bélgica, por su parte, los CP se desarrollaron antes de la legalización de MMA $(2002)^{56}$ y, con esta, CP y MMA, se desarrollaron interdependientemente bajo el modelo de "Cuidados Paliativos Integrales"57. Destaca que, según un reporte comparativo en 1999, tanto Bélgica como Países Bajos contaban con un desarrollo de CP inferior al Reino Unido, país que ha sido considerado como el estándar de referencia en esta materia ${ }^{58}$.

En 2011, la EAPC elaboró un informe comparando los datos provenientes del Atlas de Cuidados Paliativos de 2007 y otras fuentes de siete países europeos; tres con legalización de MMA (Países Bajos, Bélgica y Suiza), tres con prohibición de la misma (Alemania, Francia y España) y el Reino Unido $^{59}$. Los datos revelaron que ambos grupos tenían niveles de desarrollo de CP similares. Si bien, los autores reconocen que el análisis podría ser prematuro para evidenciar efectos, el informe concluye que "no fue posible detectar la influencia de la legalización de la muerte asistida en el desarrollo de cuidados paliativos" (p10) ${ }^{59}$.

Un estudio similar fue publicado por Bernheim et al. en $2014^{29}$. Utilizando los mismos datos provenientes del Atlas de Cuidados Paliativos, pero analizados con diferente metodología, los autores reportan conclusiones similares a las del informe EAPC previamente descrito. Los investigadores 
comparan Bélgica y Países Bajos con 4 países sin legalización de MMA (Alemania, España, Francia y Reino Unido). Considerando un ranking para cada indicador de desarrollo de CP, los autores generan un índice global para cada país. Según este índice global, luego de Reino Unido, le siguen Bélgica y Países Bajos.

Por otra parte, Chambaere y Bernheim ${ }^{27}$, publicaron un estudio comparativo en el tiempo evaluando si la legalización de MMA impactó negativamente en el desarrollo de CP. Para esto, los autores utilizaron los datos de los Atlas de Cuidados Paliativos de 2007 y 2014, considerando los siete países líderes en recursos per cápita de CP (Islandia, Bélgica, Países Bajos, Luxemburgo, Reino Unido, Suecia e Irlanda). Al comparar la variación en el tiempo, se observó un desarrollo positivo en el número total de recursos de $\mathrm{CP}$ en los siete países estudiados, con las cifras más altas para Luxemburgo, Irlanda y Países Bajos. Esto llevó a los autores a concluir que "la legalización parece haber promovido la expansión de los CP" (p. 1) ${ }^{27}$. Si bien la significación estadística de estas variaciones no se testeó para cada país, ni con respecto a otros países, los datos sugieren que la legalización de MMA en Bélgica, Países Bajos y Luxemburgo no parece estar afectando negativamente el desarrollo de CP en esos países.

La evidencia aquí expuesta presenta ciertas limitaciones a considerar; (i) la precisión de los datos de los atlas de la EAPC sobre el desarrollo de CP en cada país es cuestionable, ya que estos se obtuvieron principalmente mediante la recopilación de literatura e información aportada por "personas clave" en el campo de CP de cada país, imponiendo limitaciones respecto a la validez de los datos; (ii) algunos de los indicadores considerados en estos estudios, como la cantidad de servicios de CP por millón de habitantes, no necesariamente reflejan la calidad de la provisión de la atención paliativa y la comparación de estos indicadores es difícil entre países dada la variabilidad en los modelos de servicios locales; (iii) es preciso considerar que correlación no implica causalidad, y la metodología de los reportes aquí expuestos no permite concluir con certeza que la legalización de MMA provocó una mejoría en el desarrollo de $\mathrm{CP}$ de estos países y (iv) para efectos de este trabajo, la inclusión de los siete países líderes en el provisión de CP limita la transferibilidad de las conclusiones a países con niveles más bajos de desarrollo de CP.
Finalmente, si bien la experiencia de Colombia podría ser de gran utilidad para el contexto chileno, la revisión literaria realizada por las autoras no encontró evidencia disponible relacionada al impacto de esta legislación en el desarrollo de los CP en ese país. Tras la legalización en 1997, recién en la Ley 1733 de 2014 se establece el marco legal para los CP en Colombia ${ }^{60}$. La legalización se dio entonces, al menos, en un contexto de provisión aislada de CP (según la clasificación de la EAPC 2014, 17 años después de la legalización), realidad que ha sido también planteada en la literatura colombiana $^{49,61,62}$.

\section{Conclusiones}

En el debate sobre legalización de MMA, además de un análisis filosófico-moral de los argumentos que apoyarían o rechazarían esta práctica, es fundamental considerar los efectos que la legislación tendrá a nivel social. El derecho a una decisión autónoma debe ponderarse frente a otros intereses sociales relevantes. En relación con esto, uno de los aspectos discutidos es el potencial impacto negativo de la legalización de MMA en el desarrollo de CP.

Según lo expuesto, hasta la fecha, la evidencia disponible no respalda esta preocupación, ya que en países con legalización de MMA no se ha observado un impacto negativo en los CP. La interpretación de la evidencia requiere prudencia, dada la validez limitada de los datos disponibles y que la generalización de las conclusiones está restringida. Se necesitan estudios adicionales, con diseños prospectivos y colaboración internacional, para facilitar la comparación entre diferentes legislaciones. Además, es necesario complementarlos con investigación cualitativa que explore, entre otros, cómo pacientes, familiares y profesionales de la salud experimentan la interrelación entre MMA y CP.

La consideración de este aspecto es particularmente relevante en el contexto chileno, donde actualmente la provisión de CP es subóptima y restringida solo al grupo de pacientes oncológicos. Un proyecto responsable sobre legalización de MMA debe contemplar un compromiso real de garantizar el mínimo esencial de CP universales y de fortalecer el desarrollo y la calidad de los cuidados de final de vida. 


\section{Referencias}

1. Lampert Grassi MP. Eutanasia en la Legislación Chilena. Asesoría Técnica Parlamentaria. Biblioteca Congreso Nacional. 2019 Disponible en: https://obtienearchivo. bcn.cl/obtienearchivo?id=repositorio/10221/27133/1/ BCN_eutanasia_en_la_legislacion_chilena_FINAL.pdf [Consultado 21 de agosto de 2019].

2. Cadem. Encuesta Plaza Pública. Cadem; 2018 Disponible en: https://www.cadem.cl/encuestas/ [Consultado 21 de agosto de 2019].

3. Colegio Médico de Chile. "Encuesta de opinión acerca de la muerte asistida médicamente [Internet]. Colegio Médico de Chile. 2019. Disponible en: http://www. colegiomedico.cl/wp-content/uploads/2019/04/Resumen-Encuesta-Eutanasia.pdf

4. Rodríguez-Soriano RI. Elementos políticos para el debate sobre los dilemas éticos del suicidio médicamente asistido y de la eutanasia. Rev Ciencias la Salud [Internet] 2015; 13 (3): 465-79. Disponible en: http://revistas. urosario.edu.co/index.php/revsalud/article/view/4361.

5. Figueroa R. Matar, dejar morir y eutanasia en el proyecto de ley sobre derechos de las personas y en la doctruna chilena. Rev Med Chile 2011; 139: 655-9.

6. Kottow M. Eutanasia y Res publica. Rev Chil Salud Publica 2005; 9 (2): 111-7.

7. Carrasco M. VH, Crispi F. Eutanasia en Chile: Una discusión pendiente. Rev Med Chile 2016; 144 (12): 1598-604.

8. Vivanco Martínez A. La Autonomía de la persona frente al derecho a la vida no incluye el derecho a ser muerto por un tercero: La solicitud de asistencia al suicidio y el caso de Diane Pretty. Acta Bioeth. 2002; 2.

9. Chomalí Garib F. Morir con dignidad: el Magisterio de la Iglesia. Ars Medica Rev Ciencias Médicas 2016; 29 (1): 53.

10. Duclos HJ. Cartas al Editor: Definiciones en medicina: ¿Estamos en que el fin justifica los medios y actos médicos? Rev Med Chile 2000; 128 (7): 1-2.

11. Miranda AM. [Euthanasia, assisted suicide, and the principle of double effect: a reply to Rodolfo Figueroa]. Rev Med Chile [Internet] 2012; 140: 261-5. Disponible en: http://www.ncbi.nlm.nih.gov/pubmed/22739958.

12. Grupo de estudios de ética clínica de la Sociedad Médica de Santiago. Eutanasia y acto médico. Rev Med Chile [Internet] 2011; 139 (5): 642-54. Disponible en: http:// www.scielo.cl/pdf/rmc/v139n5/art13.pdf.

13. de la Cruz Garrido J. Eutanasia y el clima moral para su discusión en Chile. Doc Análisis, Cent Políticas Públicas, Fac Gob UDD 2017; 15: 1-15.

14. Luna F, Van Delden JM. J. Is physician-assisted death only for developed countries? Latin America as a case study. J Palliat Care [Internet] 2004; 20 (3): 155-62. Disponible en: http://pesquisa.bvsalud.org/portal/resource/ en/mdl-15511034.

15. Macklin R. Which way down the slippery slope? Nazi medical killing and euthanasia today. En: Harris J, editor. Bioethics. Forst. Oxford: Oxford University Press; 2001. p. 109-30.

16. Dzeng E. Can Growing Popular Support for Physician-Assisted Death Motivate Organized Medicine to Improve End-of-Life Care? J Gen Intern Med. 2018;1-3.

17. Leiva López A. La regulación de la eutanasia, según la ley $\mathrm{N}^{\circ} 20.584$ sobre derechos del paciente. Revista de Derecho (Valparaíso). 2013. 505-58 p.

18. Herring J. Dying and death. En: Herring J, editor. Medical Law and Ethics. Fifth. Oxford: Oxford University Press; 2014. p. 475-562.

19. Sulmasy DP, Finlay I, Fitzgerald F, Foley K, Payne R, Siegler M. Physician-Assisted Suicide: Why Neutrality by Organized Medicine Is Neither Neutral Nor Appropriate. J Gen Intern Med [Internet]. 2018; Disponible en: http://link.springer.com/10.1007/s11606-018-44248.

20. Thomasma DC. Euthanasia and assisted suicide. Cambridge Q Healthc Ethics 1998; 7: 388-401.

21. Roy DJ. Euthanasia and witholding treatment. En: Doyle D, Hanks G, Cherny N, Calman K, editores. Oxford Textbook of Palliative Medicine. Third. Oxford; 2005. p. 84-97.

22. Emanuel EJ, Onwuteaka-Philipsen BD, Urwin JW, Cohen J. Attitudes and Practices of Euthanasia and Physician-Assisted Suicide in the United States, Canada, and Europe. JAMA [Internet] 2016; 316 (1): 79-90.

23. Clark D. To Comfort Always. First Edit. Clark D, editor. Oxford: Oxford University Press; 2016.

24. Gillon R. Philosophical Medical Ethics. Fourth Edi. Gillon R, editor. Wiltshire: Wiley. British Medical Journal; 1985.

25. Simón Lorda P, Barrio Cantalejo IM, Alarcos Martínez FJ, Barbero Gutiérrez J, Couceiro A, Hernando Robles P. Ética y muerte digna: Propuesta de consenso sobre un uso correcto de las palabras. Rev Calid Asist 2008; 23 (6): 271-85.

26. Radbruch L, Leget C, Bahr P, Müller-Busch C, Ellershaw J, De Conno F, et al. Euthanasia and physician-assisted suicide: A white paper from the European Association for Palliative Care. Palliat Med 2016; 30 (2): 104-16.

27. Chambaere K, Bernheim JL. Does legal physician-assisted dying impede development of palliative care? The Belgian and Benelux experience. J Med Ethics 2015; 41 (8): 657-60. 
28. Gamondi C, Fusi-Schmidhauser T, Oriani A, Payne S, Preston N. Family members' experiences of assisted dying: A systematic literature review with thematic synthesis. Palliat Med. 2019.

29. Bernheim JL, Chambaere K, Theuns P, Deliens L. State of Palliative Care Development in European Countries with and without Legally Regulated Physician-Assisted Dying. Health Care (Don Mills) [Internet] 2014; 2 (1): 10. Disponible en: http://www.sciknow.org/article/ detail/id/1041.

30. Rodgers M, Booth A, Norman G, Sowden A. Research priorities relating to the debate on assisted dying: What do we still need to know? Results of a modified Delphi technique. BMJ Open. 2016;6(6):1-10.

31. Quill TE, Cassel CK, Meier DE. Care of the hopelessly ill. Proposed clinical criteria for physician-assisted suicide. N Engl J Med 1992; 327 (19): 1380-4.

32. Carrasco M, Hugo V, Crispi G. Eutanasia activa, una mirada a la situación internacional. Rev Hosp Clin Univ Chile [Internet] 2015; 26: 322-8. Disponible en: https:// www.redclinica.cl/Portals/0/Users/014/14/14/678.pdf.

33. Rachels J. Euthanasia: Active and passive euthanasia. N Engl J Med. 1975; 292: 78-80.

34. Organización Mundial de la Salud. Cuidados Paliativos. 2002 Disponible en: https://www.who.int/es/newsroom/fact-sheets/detail/palliative-care [Consultado 21 de agosto de 2019].

35. Connor SR, Sepulveda Bermedo MC. Global atlas of palliative care at the end of life. World Health Organization. 2014. 111 p.

36. Biblioteca del Congreso Nacional de Chile. Ley Núm. 20.584. Regula los derechos y deberes que tienen las personas en relación con acciones vinculadas a su atención en salud. 2012.

37. Zúñiga Fajuri A. Derechos Del Paciente Y Eutanasia En Chile. Rev Derecho 2008; 21 (2): 111-30.

38. Rodolfo Figueroa G. Informed consent in the patients' rights law. Rev Med Chile 2012; 140 (10): 1347-51.

39. Zúñiga Fajuri A. La nueva Ley de Derechos del Paciente: Cambiando el paradigma de la relación entre el paciente. Anu Derecho Público UDP [Internet] 2012; 273-88. Disponible en: http://www.udp.cl/descargas/ facultades_carreras/derecho/pdf/anuario/2012/Anuario_Derecho_Publico_2012.pdf.

40. Bossert TJ, Leisewitz T. Innovation and Change in the Chilean Health System. N Engl J Med [Internet] 2016; 374 (1): 1-5. Disponible en: http://www.nejm.org/ doi/10.1056/NEJMp1514202.

41. Ministerio de salud (MINSAL). Listado de prestaciones especificas. Vol. 1, Listado de prestaciones específicas GES. 2016.
42. Cámara de Diputados de Chile. Proyecto de Ley: Reconocimiento y protección de los derechos de las personas con enfermedades terminales, y el buen morir [Internet]. Proyectos de Ley 2019 [citado el 6 de septiembre de 2019]. Disponible en: https://www. camara.cl/pley/pley_detalle.aspx?prmID=13038\&prmBOLETIN=12507-11.

43. Olavarría Gambi M. Acceso a la Salud en Chile. Acta Bioeth 2005; 11 (1): 47-64.

44. Itriago GL, Silva IN, Cortés FG. Cáncer en Chile y el mundo: Una mirada epidemiológica, presente y futuro. Rev Médica Clínica Las Condes. 2013; 24 (4): 531-52.

45. Instituto Nacional de Estadisticas (INE). CHILE: Proyecciones y Estimaciones de Población, Total País 1950-2050. Inst Nac Estad. 2006;103.

46. Jansen-Van Der Weide MC, Onwuteaka-Philipsen BD, Van Der Wal G. Requests for euthanasia and physician-assisted suicide and the availability and application of palliative options. Palliat Support Care 2006; 4 (4): 399-406.

47. Van Den Block L, Deschepper R, Bilsen J, Bossuyt N, Van Casteren V, Deliens L. Euthanasia and other end of life decisions and care provided in final three months of life: Nationwide retrospective study in Belgium. BMJ 2009; 339 (7717): 390.

48. National Academy of Sciences Engineering and Medicine. 5. Implementation and Practice of Physician-Assisted Death. En: The National Academies Press, editor. Physician-assisted death: Scanning the landscape: Proceedings of a workshop [Internet]. Washington, DC; 2018. p. 1-179. Disponible en: https://www.nap.edu/ catalog/25131.

49. Mendoza-Villa JM, Herrera-Morales LA. Reflexiones acerca de la eutanasia en Colombia. Rev Colomb Anestesiol 2016; 44 (4): 324-9.

50. Hope T, Savulescu J, Hendrick J. Ethical and Legal Background. En: Hope T, Savulescu J, Hendrick J, editores. Medical Ethics and Law The core curriculum. Second. Philadelphia: Elsevier Ltd; 2008. p. 3-21.

51. Materstvedt LJ. Palliative Care ethics: the problems of combining palliation and assisted dying. Prog Palliat Care 2013; 21 (3): 158-64.

52. Gill MB. Is the Legalization of Physician-Assisted Suicide Compatible with Good End-of-Life Care? J Appl Philos 2009; 26 (1): 27-45.

53. George RJD, Finlay IG, Jeffrey D. Legalised euthanasia will violate the rights of vulnerable patients. BMJ [Internet] 2005; 331 (7518): 684-5. Disponible en: http:// www.pubmedcentral.nih.gov/articlerender.fcgi?arti$\mathrm{d}=1226254 \&$ tool $=$ pmcentrez\&rendertype $=$ abstract.

54. Barutta J, Vollmann J. Physician-assisted death 
with limited access to palliative care. J Med Ethics. 2015;41(8):652-4.

55. Janssens RJPA, Ten Have HAMJ, Zylicz Z. Hospice and euthanasia in the Netherlands: An ethical point of view. J Med Ethics 1999; 25 (5): 408-12.

56. Bernheim JL, Deschepper R, Distelmans W, Mullie A, Bilsen J, Deliens L. Development of palliative care and legalisation of euthanasia: antagonism or synergy? BMJ [Internet] 2008; 336 (7649): 864-7. Disponible en: http://www.bmj.com/cgi/doi/10.1136/ bmj.39497.397257.AD.

57. Bernheim JL, Raus K. Euthanasia embedded in palliative care. Responses to essentialistic criticisms of the Belgian model of integral end-of-life care. J Med Ethics 2017; 43 (8): 489-94.

58. Clark D, ten Have H, Janssens R. Common threads? Palliative care service developments in seven European countries. Palliat Med 2000; 14 (6): 479-90.

59. Chambaere K, Centeno C, Hernandez EA, Van Wesemael Y, Guillén-Grima F, Deliens L, et al. Palliative Care Development in Countries with a Euthanasia Law. Report for the UK Commission on Assisted Dying Briefing Papers. 2011.

60. Congreso de la República C. Ley 1733 [Internet]. 9/5/2019 Leyes desde 1992 - Vigencia expresa y control de constitucionalidad 2014 p. 1-32. Disponible en: http://www.secretariasenado.gov.co/senado/basedoc/ ley_1733_2014.html

61. Aristizábal Franco LE. Eutanasia hoy: precisiones y dudas. Rev Cult del Cuid Enfermería [Internet]. 2015; 12 (1): 83-91.

62. Díaz Amado E. La despenalización de la eutanasia en Colombia: contexto, bases y críticas. Rev Bioética y Derecho, Univ Bercelona 2004; (40): 125-40. 